\title{
Dimples and heat transfer efficiency
}

\begin{abstract}
A short review was conducted with regards to functions of dimple structures to increase the heat transfer efficiency. Dimples are among the important structure in surface texturing used to improve the parts performance such heat transfer efficiency. A review of the results of previous research found that dimple surfaced can enhance the heat transfer rate by up to $150 \%$ compared to smooth surfaces, and in certain cases the heat transfer rate is about 2.5 times higher than that of smooth surfaces. Therefore, it is believed that the dimple structure can help to enhance the product performance in various applications especially involved high temperature service conditions.
\end{abstract}

Keywords: dimple structure, heat transfer efficiency
Volume 2 Issue 5 - 2018

\author{
Jaharah A Ghani, ${ }^{1,2}$ Mohd Naqib Bin Derani,' \\ Wan Mohd Faizal Wan Mahmood 1,2 \\ 'Mechanical Engineering Programme, Malaysia \\ ${ }^{2}$ Centre for Engineering Material and Smart Manufacturing \\ (MERCU), Universiti Kebangsaan Malaysia, Malaysia
}

\author{
Correspondence: Jaharah A Ghani, Centre for Engineering \\ Material and Smart Manufacturing (MERCU), Universiti \\ Kebangsaan Malaysia, 43000 Bangi, Selangor, Malaysia, Tel \\ +60389216505, Fax +60389259659. \\ Email jaharahaggani@ukm.edu.my
}

Received: January 29, 2018 | Published: October 31, 2018

\section{Introduction}

Dimple surface is one of the surface textures that are widely studied today. In general, dimples are already well known from golf ball aerodynamics. In the case of golf balls, the application of dimples is a special form of surface roughness, which shifts the typical dropdown of the flow resistance for blunt bodies into the low Reynolds number range. Initially, the idea was to use dimples for drag reduction.

The surface functions are also controlled by the micro/nano-scale surface structures manufactured artificially such as micro-grooves and micro-dimple for industrial uses. This is because surface texture has emerged as a viable option for surface engineering, resulting in significant improvements in friction coefficient, wear resistance, load capacity, etc.

It is a well-known device for significantly enhancing heat transfer rate (generally measured in terms of Nusselt number) with possibly minimum pressure drop penalties. Belenkiy et al. ${ }^{1}$ showed that there is $150 \%$ enhancement of heat transfer rate compared to smooth surfaces while Chyu et $\mathrm{al}^{2}{ }^{2}$ reported that the heat transfer rate is about 2.5 times higher than that of a smooth surface, and the pressure losses are about half that produced by conventional rib turbulators.

The enhancement of heat transfer efficiency is needed for a variety of practical applications such as cooling electronic components, heat exchangers, cooling along the micro-fluidic passages, in the internal cooling passages of turbine blades, and even biomedical devices in continuous operation.

This article presents a mini review about the application of dimple structure for increasing the heat transfer efficiency form the previous researcher work.

\section{Previous study regarding the application of dimple structure in heat transfer}

A lot of research has been carried out on various heat transfer augmenters such as pin fins, louvered fins, offset strip fins, slit fins, ribs, protrusions and dimples in order to improve the thermal efficiency of heat exchangers. However, over past few years, dimples have received much more attention for enhancing heat transfer in internal cooling passages. This is because the previous research works have shown that dimples can enhance heat transfer in confined channels with relatively low pressure loss penalty compared to other types of augmented heat transfer devices. Ligrani et al. ${ }^{3}$ has compared several types of heat transfer augmentation techniques and concluded that dimpled surface shows a high heat transfer capacity with relatively low pressure loss penalty compared to other types of heat transfer augmenters that are available. According to Ligrani et al., the central and edge vortex pairs which are periodically shed from each dimple (as well as the resulting shear layer reattachments, boundary layer re-initialisations, and induced local unsteadiness) are key ingredients in augmenting local and spatially-averaged turbulence transport levels, and the associated surface heat transfer rates. Unlike protruding turbulators, heat transfer on dimpled surfaces is enhanced because vortex structures promote mixing, drawing "cold" fluid from outside the thermal boundary layer into contact with the wall, enhancing convective heat transfer. Therefore, a lot of research has been conducted to determine the pressure loss and heat-transfer characteristics that are caused by the dimpled surface. Research on dimpled surfaces is summarised below.

Moon et al. ${ }^{4}$ experimented on the enhancement of heat transfer using a convex-patterned surface. Moon et al. conclude that enhancement of heat transfer using a convex patterned surface was thermally more effective at relatively low Reynolds numbers than that of a smooth channel. Meanwhile Silva et al. ${ }^{5,6}$ investigated the flow structure and heat transfer enhancement of rectangular mini channel with dimples on one side of the wall. The results showed that the drag coefficient and heat transfer were increased to some extent. Wei et al. ${ }^{7}$ $\&$ Lan et al. ${ }^{8}$ studied the flow and heat transfer enhancement of microchannel with dimple. However, the misuse of boundary condition may lead to some discrepancies between the simulated results and real flow in the experiment conducted by Wei et al. The studies showed that the application of dimple and protrusion in micro-channel heat sinks could obtain low-pressure penalty and higher heat transfer coefficient in laminar regime. ${ }^{9}$

Recently, Suresh et al..$^{10,11}$ experimentally studied the flow and heat transfer of $\mathrm{CuO}$-water nanofluids in the channel with dimple in laminar and turbulent regime. Results show that the use of nanofluids in a helically dimpled tube increases the heat transfer rate. Experiments done by Griffith et al. ${ }^{12}$; Kim et al. ${ }^{13}$ in rotating rectangular channels describe that rotation could increase the heat transfer coefficient on dimpled surfaces, especially on the trailing dimpled surface, making it higher than that on the leading surface. 
Mahmood \& Ligrani $^{14}$ conducted research to expose the characteristics of the flow structure induced by the dimpled surface on one side wall of a channel with three different channel heights $(\mathrm{H} / \mathrm{d}=0.25,0.50$, and 1.00) in the range of $\mathrm{ReH}=600-11,000$. The visualization of the flow results showed the shedding of vertical structures from the dimple cavity. The maximum Nussel number appeared on the dimple rim and the flat surface adjacent to the downstream of each dimple. The minimum heat transfer was observed in the flow-recirculation region inside the dimple. Through experiments, Burgess et al. ${ }^{15}$ showed the influence of the dimple depth and the dimple imprint diameter on heat transfer, and found that the local and spatially-averaged Nusselt number augmentations increase with an increase in the dimple depth, for various Reynolds numbers from 12,000 to 70,000 . Doo et al. ${ }^{16}$ conducted a parametric study on flows passing through a channel with newly designed surface shapes that comprised of a combination of dimple and riblet. The superior thermo-aerodynamic performance, assessed in terms of the volume goodness factor, was predicted in the riblet-mounted dimple case with a riblet angle of $60^{\circ}$.

Experiments by Xiao et al. ${ }^{17}$ show that thermal performance parameters were higher in a heat exchanger with a dimpled bottom and smooth top than in a heat exchanger with a dimpled bottom and protrusions on top in laminar region, and proposed friction factor ratio and Nusselt number ratio correlations for a heat exchanger with dimpled bottom and smooth top. Burgess et al. ${ }^{15}$; Ligrani ${ }^{18}$ experimentally investigated the characteristics of the friction factor and Nusselt number with respect to dimple depth. Both proposed a Nusselt number ratio correlation as a function of the dimple print diameter and dimple depth in a heat exchanger with dimples on the bottom and a smooth top.

Shape optimization studies for dimpled plate heat exchangers have also been conducted. ${ }^{19,20} \mathrm{Choi}^{19} \& \mathrm{Kim}, \mathrm{Shin}^{20}$ carried out multiobjective optimization for a heat exchanger with a dimpled bottom and smooth top, using approximate models obtained via the response surface method and the Kriging method, respectively. Kim et al. ${ }^{21}$ generated a Pareto optimal solution in staggered elliptic dimpled channels, using an evolutionary multi-objective algorithm with the Kriging method.

\section{Conclusion}

Dimple surfaces can enhance the heat transfer rate by up to $150 \%$ compared to smooth surfaces, and in certain cases the heat transfer rate is about 2.5 times higher than that of smooth surfaces.

\section{Acknowledgments}

None.

\section{Conflict of interest}

The author declares that there is no conflict of interest.

\section{References}

1. Belenkiy M, Gotovskiy M, Lekakh B, et al. Heat transfer augmentation using surfaces formed by a system of spherical cavities. Heat Transfer Research. 1993;25(2):196-203.

2. Chyu MK, Yu Y, Ding H, et al. "Concavity Enhanced Heat Transfer in an Internal Cooling Passage”. ASME. 1997:437.
3. Ligrani PM, Oliveira MM, Blaskovich T, et al. Comparison of heat transfer augmentation techniques. AIAA J. 2003;41(3):337-372.

4. Moon HK, Connell TO, Sharma B, et al. Heat transfer enhancement using a convex patterned surface. ASME J Heat Transfer. 2003;125(2):274-280.

5. Silva C, Marotta E, Fletcher L, et al. Flow structure and enhanced heat transfer in channel flow with dimpled surfaces: application to heat sinks in microelectronic cooling. J Electron Packag. 2007;129(2):157-166.

6. Silva C, Park D, Marotta E, et al. "Optimization of fin performance in a laminar channel flow through dimpled surfaces". Journal of Heat Transfer. 2009;131(2):9.

7. Wei X, Joshi Y, Ligrani P, et al. Numerical simulation of laminar flow and heat transfer inside a microchannel with one dimpled surface. J Electron Packag. 2007;129(1):63-70.

8. Lan JB, Xie YH, Zhang D, et al. Flow and Heat Transfer in Microchannels with Dimples and Protrusions. Journal of Heat transfer. 2012;134(2):9.

9. Bruzzone AAG, Costa HL, Lonardo PM, et al. Advances in engineered surfaces for functional performance. CIRP Annals- Manufacturing Technology. 2008;57(2):750-769.

10. Suresh S, Chandrasekar M, Chandra Sekhar S, et al. Experimental studies on heat transfer and friction factor characteristics of $\mathrm{CuO} /$ water nanofluid under turbulent flow in a helically dimpled tube. Exp Therm Fluid Sci. 2011;35(3):542-549.

11. Suresh S, Chandrasekar M, Selvakumar P, et al. Experimental studies on heat transfer and friction factor characteristics of $\mathrm{CuO} /$ water nanofluid under laminar flow in a helically dimpled tube. Heat Mass Transfer. 2012;48(4):683-694.

12. Griffith TS, Al-Hadhrami L, Han JC, et al. Heat transfer in rotating rectangular cooling channels with dimples. ASME J Turbomach. 2003;125(3):555-564.

13. Kim S, Choi EY, Kwak JS, et al. Effect of channel orientation on the heat transfer coefficient in the smooth and dimpled rotating rectangular channels. ASME J Heat Transfer. 2012;134(6):5.

14. Mahmood GI, Ligrani PM. Heat transfer in a dimpled channel: combined influences of aspect ratio temperature Reynolds number and flow structure Temperature Reynolds Number and Flow Structure. Int J Heat Mass Transfer. 2002;45(10):2011-2020.

15. Burgess NK, Oliveira MM, Ligrani PM, et al. Nusselt number behavior on deep dimpled surfaces within a channel. ASME Trans J Heat Transfer. 2003;125(1):11-18.

16. Doo JH, Yoon HS, Ha MY, et al. Study on improvement of compactness of a plate heat exchanger using a newly designed primary surface. Int J Heat Mass Transfer. 2010;53(26):5733-5746.

17. Xiao N, Zhang Q, Ligrani PM, et al. Thermal performance of dimpled surfaces in laminar flows. Int J Heat Mass Transfer. 2009;52(8):20092017.

18. Burgess NK, Ligrani PM. Effects of dimple depth on channel Nusselt numbers and friction factors. J Heat Transfer. 2005;127(8):839-847.

19. Kim KY, Choi JY. Shape optimization of a dimpled channel to enhance turbulent heat transfer. Numer Heat Transfer A Appl. 2005;48(9):901-915.

20. Kim KY, Shin DY. Optimization of a staggered dimpled surface in a cooling channel using Kriging model. Int J Therm Sci. 2008;47(11):1464-1472.

21. Kim HM, Moon MA, Kim KY, et al. Multi objective optimization of a cooling channel with staggered elliptic dimples. Energy 2011;36(5):34193428 . 